\title{
Evaluación de la incertidumbre en la determinación de aflatoxina B1 en maní de exportación por HPLC-FD. Parte I
}

\author{
Gustavo Delgado ${ }^{*}$, Norma Hernández $^{2 *}$
}

1. Universidad Nacional Autónoma de Nicaragua, León (UNAN-LEÓN). Facultad de Ciencias y Tecnología, Departamento de Química, Laboratorio de Análisis de Trazas de Metales Pesados (LATMP), Edificio de Ciencias Básicas, León, Nicaragua. Tel: (505) 2311 5013, ext: 1132. Fax: (505) 23114012.

2. Laboratorio SER-AGRO S.A., Km 38 carretera Chinandega-Corinto, Tel: 23403493.

\section{RESUMEN}

El presente trabajo tiene como principal objetivo estimar la incertidumbre en la determinación de aflatoxina B1 en una muestra de maní de exportación por Cromatografía Líquida de Alto Desempeño con Detector de Fluorescencia (HPLC-FD). El cálculo se basó en el procedimiento general establecido en las guías para la expresión de la incertidumbre GUM ISO 1995 y EURACHEM/CITAC. Se identificaron 18 variables del modelo matemático que influyen en la incertidumbre del contenido de aflatoxina B1 en la muestra de maní. El valor de la incertidumbre combinada, calculada por la ley de propagación de la incertidumbre, fue de 0.224632 y el contenido de aflatoxina B1 fue de $1.47 \mathrm{ng} / \mathrm{g}$. Se evaluaron los índices de contribución de cada variable a la incertidumbre combinada y se demostró a través del diagrama de Pareto, que solamente cinco variables son las que influyen significativamente en la incertidumbre de los resultados, calculándose un valor de 0.223492. El intervalo de confianza para una muestra de maní fue $(1.47 \pm 0.45) \mathrm{ng} / \mathrm{g}$ para un factor de cobertura de 2 al $95.45 \%$ de nivel de confianza. La incertidumbre estándar relativa, calculada con la máxima precisión, fue de $15 \%$, siendo aceptable a los niveles de concentración del presente estudio.

Palabras claves: incertidumbre estándar relativa, aflatoxina B1 por HPLC en maní, coeficiente de sensibilidad, Maple.

\section{INTRODUCCIÓN}

La presencia de aflatoxinas B1, B2, G1 y G2 en cereales se debe a las micotoxinas producidas por el hongo aspergillus flavus ${ }^{[1]}$. Se dice que todas estas micotoxinas son peligrosas para la salud. Se ha demostrado que la aflatoxina B1 produce cáncer en el hígado ${ }^{[2]}$. Cuando el maní no es almacenado en condiciones ambientales apropiadas (mucha húmedad y altas temperaturas) ${ }^{[1]}$ se favorece el crecimiento de los hongos.

El nivel de exigencia del contenido de aflatoxinas en maní como alimento es muy variado, según el país. La FAO/OMS establece hasta $15 \mu \mathrm{g} / \mathrm{Kg}$ (o ng/g) de aflatoxinas totales ${ }^{[3]}$. Compradores de maní de países europeos como Inglaterra, exigen que la concentración máxima tolerable (CMT) de aflaxotina B1 no debe ser mayor de $1.5 \mathrm{ng} / \mathrm{g}$ y el CMT de aflatoxinas totales no debe ser mayor que $3 \mathrm{ng} / \mathrm{g}$.

El producto debe ir acompañado de un certificado de análisis de un laboratorio que tenga la competencia técnica para realizar el ensayo basado en las normas ISO/ IEC 17025:2005 y que esté acreditado por un organismo nacional de acreditación. Los resultados deben cumplir con los parámetros de calidad: trazabilidad, precisión y confiabilidad.

\footnotetext{
*Autor para correspondencia: gdelgado@unanleon.edu.ni
} nhernandez@comasa.com.ni
De esta manera, el producto lleva el informe de la conformidad, declarando el valor del mensurando (concentración de aflatoxina B1), su incertidumbre asociada y un nivel de confiabilidad especificado. Los compradores disponen de una guía donde se describe el método que tienen que cumplir los laboratorios de ensayos para declarar los resultados y la evaluación del cumplimiento con la especificación del producto dentro de los límites establecidos ${ }^{[4,5]}$ en los acuerdos comerciales. Estos límites requieren que cada laboratorio de ensayo disponga de un procedimiento documentado para evaluar la incertidumbre ${ }^{[6-10]}$ en los resultados de la medición del mensurando.

Para garantizar la calidad del maní que la empresa agroindustrial Comercializadora del Maní S. A. (COMASA) exporta a Estados Unidos e Inglaterra, el Laboratorio de Servicios Agroindustriales (SER-AGRO, S.A.) ha implantado el Sistema de la Calidad basado en la norma ISO/IEC 17025:2005 ${ }^{[11]}$, y ha acreditado el ensayo para la determinación de aflatoxinas (B1, B2, G1 y G2) por Cromatografía Líquida de Alto Desempeño (HPLC) ante la Oficina Nacional de Acreditación ONAMIFIC[12].

La evaluación de la conformidad del producto que el laboratorio extiende debe reflejar los parámetros de 
calidad de los resultados, es decir el contenido de aflatoxinas en $\mu \mathrm{g} / \mathrm{Kg}$ con su incertidumbre asociada (exactitud, precisión y confiabilidad).

En el presente estudio se pretende presentar la metodología utilizada para estimar la incertidumbre en la determinación de la concentración de aflatoxina B1 en el maní de exportación, de acuerdo a los requisitos de la norma ISO/IEC 17025:2005 (numeral 5.4.6) ${ }^{[11]}$, siguiendo las directrices generales de las guías GUM ISO $1995^{[6]}$ y EURACHEM ${ }^{[9]}$, utilizando como herramienta de cálculo el programa de cálculo simbólico MAPLE ${ }^{[13]}$.

\section{TEORÍA}

En trabajos anteriores se explicó el principio del cálculo de la incertidumbre combinada ${ }^{[10]}$, aplicando la ley de la propagación de la incertidumbre ${ }^{[6,14,15]}$. Existe también otro método para estimar la incertidumbre, la cual se basa en la Simulación de Monte Carlo (SMC) ${ }^{[16-18]}$. Esto significa que la estimación de la incertidumbre se puede calcular por dos vías, tal a como lo muestra la figura 1.

En el presente estudio se utilizó la primera vía, la aplicación de la ley de propagación de la incertidumbre. El principio del cálculo basada en el enfoque de proceso es el siguiente. Las variables de entrada son $x_{1}, x_{2}, \ldots x_{i}$, relacionadas a través de la función $f$, la variable de salida es $y$ con su incertidumbre asociada $U$, siendo $U=k \times u_{y}$.

Donde $k$ es igual a 2 para un nivel de confianza del $95.45 \%$ y $u$ es la incertidumbre combinada calculada por la expresión de propagación de la incertidumbre ${ }^{[10,14,15]}$ representada en la ecuación (1).

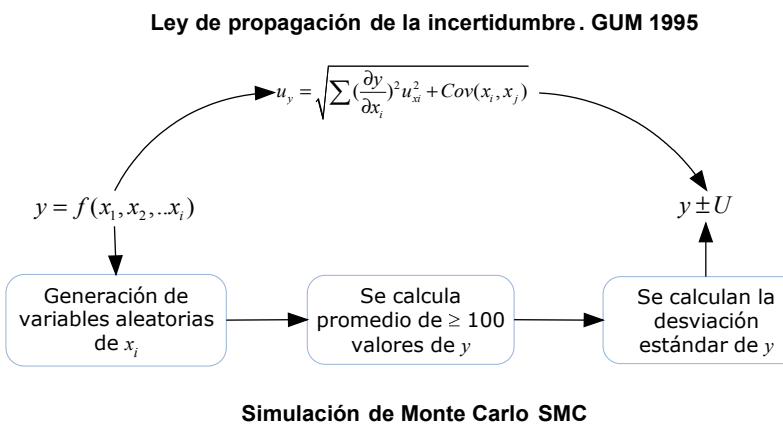

Figura 1. Estimación de la incertidumbre del mensurando y a través de dos vías: ley de propagación de la incertidumbre y por Simulación de Monte Carlo.

$$
u_{(y)}^{2}=\sum\left(\frac{\partial f}{\partial x_{i}}\right)^{2} u_{x i}^{2}+2 \sum_{i=1}^{N-1} \sum_{j=i+1}^{N}\left(\frac{\partial f}{\partial x_{i}}\right)\left(\frac{\partial f}{\partial x_{j}}\right) u_{\left(x_{i}, x_{j}\right)}
$$

\section{Procedimiento para la evaluación de la incertidumbre}

El procedimiento para estimar la incertidumbre es el utilizado en trabajos anteriores ${ }^{[10]}$. Este procedimiento se presenta en el diagrama de flujo de la figura 2.

Se define el mensurando, se deriva el modelo matemático, se identifican las fuentes de incertidumbres (las de tipo A, obtenidas a partir de la desviación estándar de una serie de observaciones y las de tipo $B$, obtenidas a partir de las distribuciones normal, rectangular 0 triangular $\left.{ }^{[6-9]}\right)$, se cuantifican las incertidumbres, se calculan los coeficientes de sensibilidad, se hace un presupuesto de las incertidumbres, se combinan las incertidumbres a través de la ley de la propagación de la incertidumbre, se revisa si no hay incongruencias

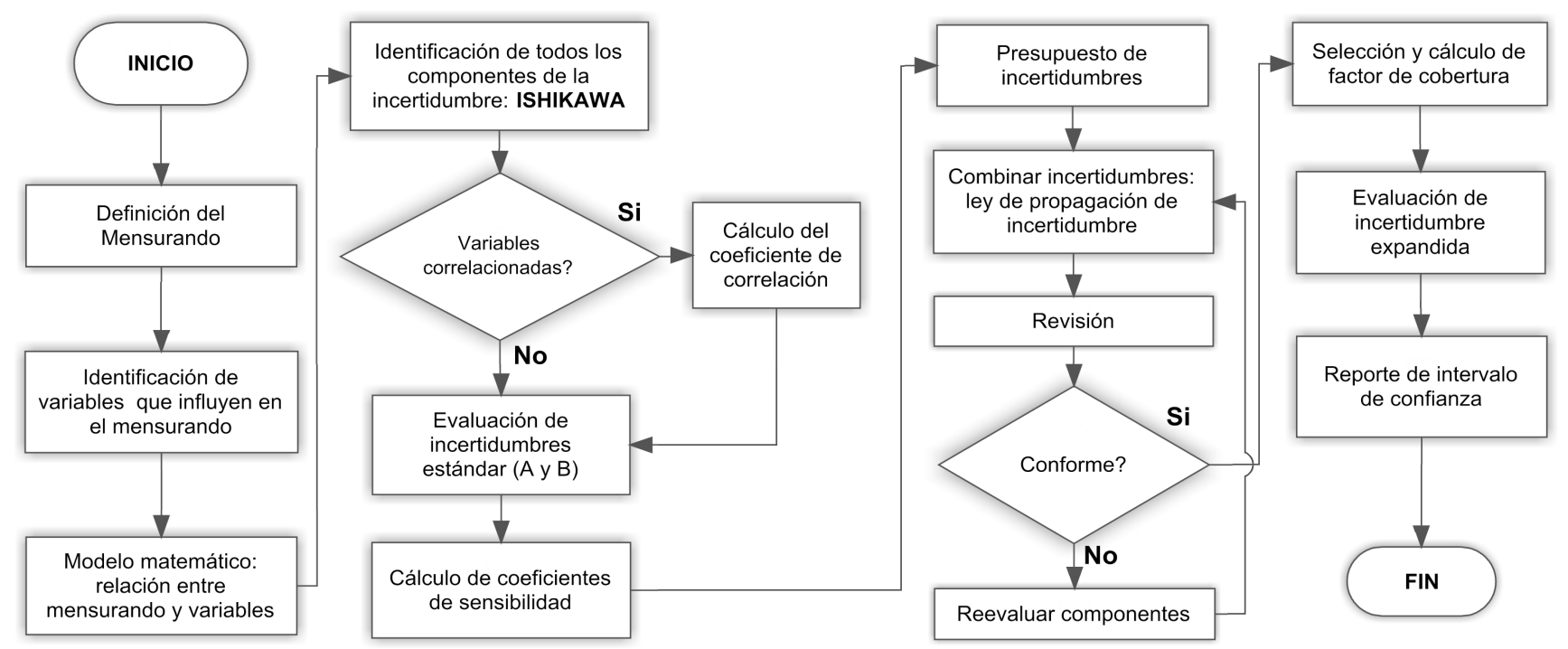

Figura 2: Diagrama de flujo representando el procedimiento para la estimación de la incertidumbre en un proceso de medición. 
para reevaluar los componentes de la incertidumbre, se selecciona el factor de cobertura, se evalúa la incertidumbre expandida y se reporta el intervalo de confianza con el número de cifras significativas correcto.

\section{METODOLOGÍA}

Todas las mediciones fueron efectuadas con los instrumentos calibrados con patrones de trabajo trazables al sistema SI y con materiales de referencia certificados. El detalle del procedimiento y las condiciones de operación del HPLC se encuentran en los documentos del laboratorio ${ }^{[19]}$.

\section{Equipos, materiales y reactivos}

El equipo utilizado es un cromatógrafo HPLC HP1050 con detector de flourescencia (FD) HP-1046A y con un reactor quimioluminiscente post columna para la reacción fotoquímica con UV de las aflaxotinas B1 y G1. Acoplado a un computador con el programa ChemStation Agilent para el registro y tratamiento de las señales.

Los materiales, reactivos y solventes están detallado en el procedimiento del laborotorio ${ }^{[19]}$. Las soluciones patrones se prepararon a partir de soluciones de referencias certificadas. La balanza (con resolución \pm $0.01 \mathrm{~g}$ ) y los volumétricos utilizados estaban calibrados, con su certificado de calibración trazable al SI. La variación de temperatura ambiental se registró con un termómetro calibrado trazable al SI.

\section{Procedimiento}

Preparación y medición del área de pico de la solución de referencia: Se tomó una alícuota de $1 \mathrm{~mL}$ de la solución de referencia certificada de aflatoxina B1 de concentración $C_{M R C}=(3.415 \pm 0.025) \mathrm{mg} / \mathrm{L}$ y se diluyó a $10 \mathrm{~mL}$ Se preparó exactamente una mezcla de 25 $\mathrm{mL}$ de agua y $25 \mathrm{~mL}$ de metanol y se le añadió $1 \mathrm{~mL}$ de la solución de referencia diluida. Se homogenizó y se inyectaron $30 \mu \mathrm{L}$ al HPLC, obteniéndose el cromatograma y registrándose el área de pico.

\section{Procedimiento para la determinación de la aflatoxina B1}

Se prepararon $100 \mathrm{~mL}$ de una mezcla acuosa de harina de maní al 33.33\%. Se añadieron $200 \mathrm{~mL}$ metanol. La mezcla resultante fue homogenizada. Se filtró, se recolectaron $30 \mathrm{~mL}$ y se le añadieron $20 \mathrm{~mL}$ de salmuera al $20 \%$. Después de mezclar se tomaron $40 \mathrm{~mL}$ y se añadieron $3.5 \mathrm{~mL}$ de cloroformo y luego se agitó. Se separó la capa orgánica de la acuosa. Se tomó una alícuota de $1.6 \mathrm{~mL}$ de la capa clorofórmica y se llevó sequedad con nitrógeno. Se restituyó el mismo volumen con metanol-agua al 80:20. La solución fue inyectada al HPLC y se realizó el proceso cromatográfico, midiendose el área de pico de acuerdo al procedimiento del laboratorio ${ }^{[19]}$.

\section{RESULTADOS Y DISCUSIÓN}

Los resultados que a continuación se presentan siguen el procedimiento descrito en el flujograma ${ }^{[10]}$ de la figura 2, basado en la guía GUM ISO $1995^{[6]}$ y la EURACHEM/ CITAC ${ }^{[9]}$.

\section{Definición del mensurando e identificación de las variables que influyen en el mensurando}

El mensurando definido para este trabajo es el contenido de aflatoxina B1 en maní de exportación simbolizado por la letra $y$ en unidades de $\mathrm{ng} / \mathrm{g}$. De acuerdo al procedimiento descrito en la metodología ${ }^{[24]}$, las variables que influyen en el mensurando se resumen en cuatro partes: a) las mediciones de las áreas de pico de los cromatogramas correspondientes a la aflatoxina B1 de la solución muestra y la de la solución de referencia diluida; b) la concentración de la aflatoxina B1 en la solución de referencia, su pureza y sus factores de dilución; c) el peso de la muestra y los factores de dilución; d) el porcentaje de recuperación. En la tabla 1 se especifican las variables con sus valores y la simbología utilizada.

\section{Modelo matemático}

En la ecuación (2) se presenta el modelo matemático deducido del proceso analítico, para medir la concentración (en $\mathrm{ng} / \mathrm{g}$ ) de aflatoxina B1 en una muestra de maní de exportación, designada por la función $f$, la cual relaciona las variables de la tabla 1, incluyendo la exactitud del método representada por el porcentaje de recuperación $(R c)$, y obtenido a partir de los datos de validación y del control de calidad del método ${ }^{[20,22]}$

$$
f=\left(\frac{A_{x}}{A_{s}}\right)\left(\frac{C_{M R C} P_{v_{0} v_{03}}}{v_{02}\left(v_{03}+v_{04}+v_{05}\right)} \times 1000\right)\left(\frac{V_{4}\left(V_{1}+V_{2}\right)\left(V_{0}+V_{s}\right) V_{6}}{V_{5} V_{3} V_{1} w}\right)\left(\frac{100}{R_{c}}\right)
$$


Tabla I: Variables que influyen en el mensurando $y$.

\begin{tabular}{cl}
\hline Variable $x_{i}$ & \multicolumn{1}{c}{ Especificación (unidades) } \\
\hline$A_{x}=12.2233$ & Área de pico de aflatoxina B1 en la solución muestra (LU-S) \\
$A_{s}=76.0983$ & Área de pico de aflatoxina B1 en la solución de referencia (LU-S) \\
$C_{M R C}=3.415 \pm 0.025$ & Concentración de aflatoxina B1 de la solución de referencia certificada $(\mathrm{mg} / \mathrm{L})$ \\
$P=99 / 100$ & Pureza de la aflatoxina B1 en la solución de referencia $(P \% / 100)$ \\
$v_{01}=1$ & Alícuota de la solución de referencia certificada $(\mathrm{mL})$ \\
$v_{02}=10$ & Volumen de dilución con metanol para la solución de referencia $(\mathrm{mL})$ \\
$v_{03}=1$ & Alícuota de la solución de referencia diluida en $v_{02}(\mathrm{~mL})$ \\
$v_{04}=25$ & Volumen de agua agregado a $v_{03}(\mathrm{~mL})$ \\
$v_{05}=25$ & Volumen de metanol agregado a $v_{04}(\mathrm{~mL})$ \\
$V_{s}=100$ & Volumen de "slurry" (mezcla acuosa) de harina de muestra de maní $(\mathrm{mL})$ \\
$V_{0}=200$ & Volumen metanol agregado a $V_{s}(\mathrm{~mL})$ \\
$V_{1}=30$ & Volumen recolectado de la filtración de $V_{0}(\mathrm{~mL})$ \\
$V_{2}=20$ & Volumen de salmuera al 20\% agregado a $V_{l}(\mathrm{~mL})$ \\
$V_{3}=40$ & Volumen recolectado de la filtración de $V_{2}+V_{1}(\mathrm{~mL})$ \\
$V_{4}=3.5$ & Volumen de cloroformo agregado a $V_{3}(\mathrm{~mL})$ \\
$V_{5}=1.6$ & Volumen de cloroformo recolectado después de separación $(\mathrm{mL})$ \\
$V_{6}=1.6$ & Volumen añadido de metanol-agua $(\mathrm{mL})$ \\
$w=33.33$ & Peso de la muestra de harina de maní $(\mathrm{g})$ \\
$R_{c}=94.78$ & Recuperación $(\%)$ \\
\hline
\end{tabular}

\section{Identificación de los componentes de la diluciones, el peso de la muestra de maní y sus incertidumbre \\ diluciones, y la recuperación.}

Los componentes de las incertidumbres para cada variable del modelo se muestran en el diagrama causaefecto de la figura 3.

Las variables se encuentran agrupadas en las cuatro partes de la ecuación 2: las lecturas de las áreas de pico de la muestra y de la solución de referencia, la concentración de la solución de referencia y sus

\section{Cuantificación de los componentes de la incertidumbre}

A continuación se presentan los cálculos de las incertidumbres de las variables del modelo. La incertidumbre de la pureza no está declarada en el certificado. Por tanto se asume como una constante.

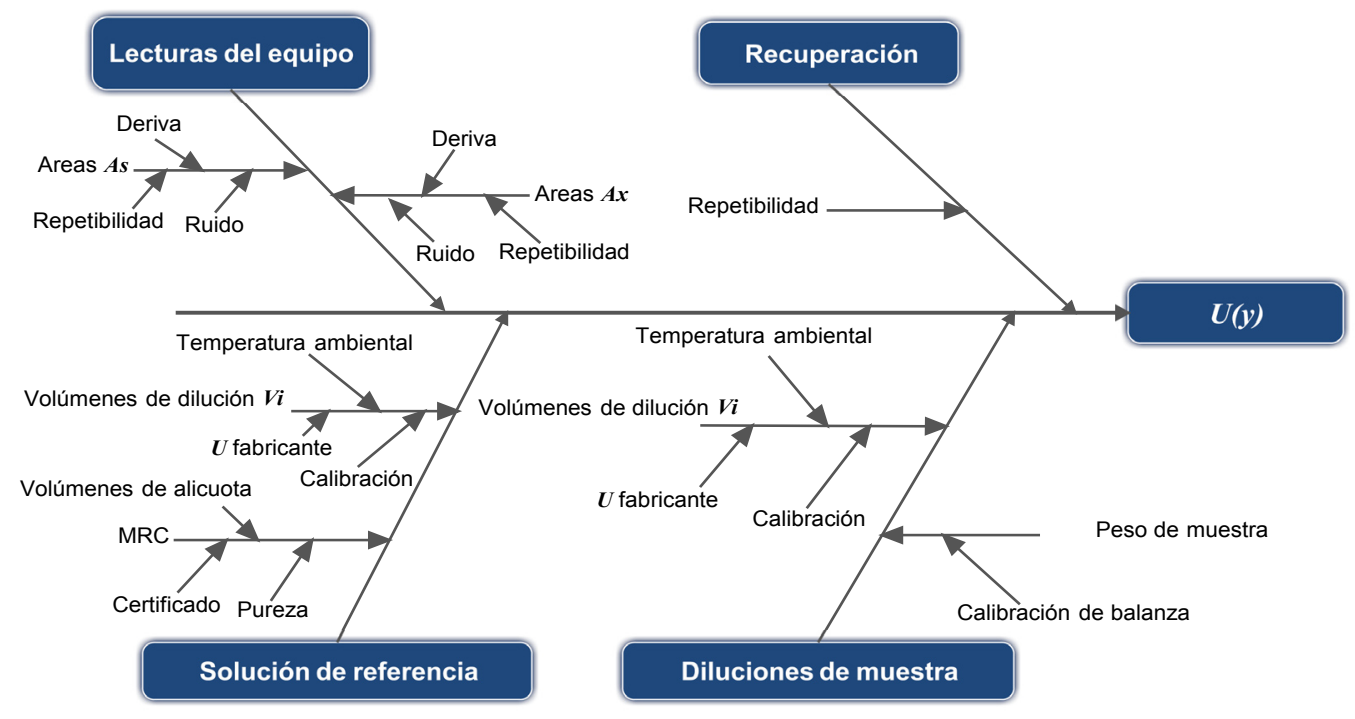

Figura 3. Diagrama causa-efecto para componentes de la incertidumbre en la medición del área de la figura geométrica del anexo. 


\section{a) Incertidumbre en las mediciones de las áreas de pico del estándar y muestra $u_{A i}$}

Para calcular la incertidumbre de las mediciones en las áreas de pico es necesario tener en cuenta tres componentes de precisión intermedia ${ }^{[21]}$ (o reproducibilidad dentro del laboratorio): las lecturas del área de pico cromatográfico, las del ruido y las de la deriva. El cálculo de la incertidumbre se realiza utilizando la ecuación (3).

$$
u_{A i}=\sqrt{\frac{s_{A}^{2}}{n_{A}}+\frac{s_{R u}^{2}}{n_{R}}+\frac{s_{d}^{2}}{n_{d}}}
$$

Donde:

- $\quad u_{A i}$ es la incertidumbre del área de pico de la solución de referencia o estándar de aflatoxina B1 $(A s)$ y la de la muestra de referencia interna cuya matriz es similar a la muestras de rutina ${ }^{[22]}\left(A_{x}\right)$;

- $s_{A}^{2}$ es la desviación estándar de las áreas de picos, leídas en condiciones de precisión intermedia $\left(n_{A}=5\right.$ mediciones realizadas diariamente durante 7 meses, por 3 analistas diferentes);

- $\quad s_{R u}^{2}$ es la desviación estándar del ruido (tomando la señal del ruido de pico a pico usando la técnica ASTM a intervalos cortos de tiempo ${ }^{[23]}$ ), calculada con una lectura diaria $\left(n_{R}=1\right)$ durante 7 meses y realizada por 3 analistas diferentes;

- $\quad s_{d}^{2}$ es la desviación estándar de la deriva calculada con una lectura diaria $\left(n_{d}=1\right)$ durante 7 meses y realizada por 3 analistas diferentes.

Los resultados se resumen en la tabla II.
Tabla II. Deviaciones estándar ${ }^{[24]}$ e incertidumbres de áreas de pico de la solución de referencia y la muestra*.

\begin{tabular}{ccccc}
\hline$A_{i}$ & $S_{A}$ & $S_{R u}$ & $S_{d}$ & $u_{A i}$ \\
\hline$A_{S}$ & $1.251(5)$ & $0.106(1)$ & $0.9469(1)$ & 1.1038 \\
$A_{X}$ & $2.529(5)$ & $0.106(1)$ & $0.9469(1)$ & 1.4782 \\
\hline
\end{tabular}

$\left(^{*}\right)$ El numero entre paréntesis indica el número de lecturas diarias $n_{i^{*}}$

\section{b) Incertidumbre en las medidas de volumen $u_{V i}$}

La incertidumbre en la medición de volumen a partir de volumétricos calibrados, se calcula tomando en cuenta tres componentes: la desviación estándar de calibración, la incertidumbre del fabricante y la incertidumbre debido al efecto que tiene la variación de la temperatura ambiental sobre la expansión de los líquidos ${ }^{[9]}$. La ecuación para el cálculo de $u_{V i}$ es la siguiente:

$$
u_{V i}=\sqrt{\frac{s_{r}^{2}}{n}+\frac{a^{2}}{6}+\frac{\left(V_{i} \alpha_{L} \Delta t\right)^{2}}{12}}
$$

Donde:

- $s_{r}$ es la desviación estándar de repetibilidad de calibración del volumétrico en $\mathrm{mL}$ (distribución $t$ ) calculada para $\mathrm{n}=10$ réplicas,

- $\quad a$ es la tolerancia en $\mathrm{mL}$ del volumétrico establecida por el fabricante (distribución triangular) ${ }^{[9]}$,

- $V_{i}$ es el volumen en $\mathrm{mL}$ medido con el volumétrico,

- $\alpha_{L}$ es el coeficiente de expansión del líquido en $1 /{ }^{\circ} \mathrm{C}^{[25]}$,

- $\Delta \mathrm{t}$ es la variación máxima de la temperatura ambiental en ${ }^{\circ} \mathrm{C}$ (distribución rectangular) ${ }^{[0]}$.

Los resultados del cálculo de las incertidumbres de los diferentes volumétricos se pueden ver en la tabla III.

Tabla III. Tabla de incertidumbres para los diferentes volumétricos y coeficientes de expansión de los solventes utilizados. La variación de temperatura del ambiente fue de $22^{\circ} \mathrm{C}$ a $28^{\circ} \mathrm{C}$.

\begin{tabular}{ccccccc}
\hline Variable & $V_{i}(\mathrm{~mL})$ & $a(\mathrm{~mL})$ & $S_{r}(\mathrm{~mL})$ & $\alpha_{L}{ }^{\circ} \mathrm{C}$ & $u_{V i}(\mathrm{~mL})$ & Solvente $^{[25]}$ \\
\hline$V_{02}$ & 10.00 & 0.02 & 0.0002957 & 0.001259 & 0.0232869 & Metanol $(\mathrm{MeOH})$ \\
$V_{05}$ & 25.00 & 0.03 & 0.0077214 & 0.001259 & 0.0564061 & $\mathrm{MeOH}$ \\
$V_{04}$ & 25.00 & 0.03 & 0.0077214 & 0.000210 & 0.0170970 & agua \\
$V_{6}$ & 1.60 & 0.05 & 0.0037274 & 0.000735 & 0.0205475 & MeOH/Agua \\
$V_{5}$ & 1.60 & 0.01 & 0.0022784 & 0.001273 & 0.0058569 & Cloroformo \\
$V_{4}$ & 3.50 & 0.02 & 0.1447340 & 0.001273 & 0.1451694 & Cloroformo \\
$V_{3}$ & 40.0 & 0.5 & 0.0480084 & 0.000770 & 0.2141368 & MeOH/Agua/salm \\
$V_{2}$ & 20.00 & 0.02 & 0.0143783 & 0.000414 & 0.0218878 & Salmuera 20\% \\
$V_{1}$ & 30.00 & 0.03 & 0.0077214 & 0.001000 & 0.0539409 & $\mathrm{MeOH} / \mathrm{Agua}$ \\
$V_{0}$ & 200 & 2 & 0.0674949 & 0.001259 & 0.9281336 & $\mathrm{MeOH}$ \\
$V_{03}$ & 1.00 & 0.14 & 0.0006779 & 0.001259 & 0.0572004 & $\mathrm{MeOH}$ \\
$V_{01}$ & 1.00 & 0.14 & 0.0006779 & 0.001259 & 0.0572004 & $\mathrm{MeOH}$ \\
$V_{s}$ & 100.00 & 0.01 & 0.0050000 & 0.000210 & 0.0369414 & Agua \\
\hline
\end{tabular}




\section{c) Incertidumbre de la concentración de la solución de referencia certificada $u C_{M R C}$}

La incertidumbre de la concentración de la solución de referencia certificada se calcula a partir del intervalo de confianza declarado en el certificado. Como no se especifica la forma en que se evaluó la incertidumbre asociada, se asume entonces que sigue una distribución rectangular ${ }^{[9]}$, dado que sólo se conocen los valores extremos.

El certificado declara que la concentración de la solución de aflatoxina B1 es:

$$
\text { (3.415 } \pm 0.025) \mathrm{mg} / \mathrm{L}
$$

La incertidumbre estándar de la concentración de la solución de referencia se obtiene dividiendo 0.025 entre la raíz cuadrada de 3 :

$$
u C_{M R C}=0.014338 \mathrm{mg} / \mathrm{L} \text {. }
$$

\section{d) Incertidumbre en el peso de la muestra de maní} $u_{w}$

La incertidumbre en el peso de la muestra de maní se calculó a partir de los datos del certificado de calibración de la balanza utilizada en el ensayo.

La resolución $(R)$ especificada por el fabricante es de $0.01 \mathrm{~g}$ y la variación máxima de la linealidad es de \pm 0.008 . Como las pesadas fueron realizadas por diferencia, ajustando el cero del recipiente de pesada, la incertidumbre de la linealidad debe multiplicarse por $2^{[9]}$.

La ecuación (5) es la fórmula para calcular la incertidumbre en la pesada de la muestra de maní.

$$
u_{w}=\sqrt{2 \frac{u_{L}^{2}}{3}+\frac{u_{R}^{2}}{12}}
$$

Donde:

- $u_{L}^{2}$ es la incertidumbre de la linealidad de calibración

- $u_{R}^{2}$ es la incertidumbre debido a la resolución especificada por el fabricante.

La resolución y la linealidad siguen una distribución rectangular. Aplicando la ecuación (5) se obtiene el valor de la incertidumbre en la pesada:

$$
u_{w}=0.0071414 \mathrm{~g}
$$

\section{Incertidumbre en el porcentaje de recuperación $u R c$}

La incertidumbre en el porcentaje de recuperación se calculó a partir de una serie de 6 ensayos realizados a una muestra de referencia interna durante 6 meses (un ensayo por mes). La desviación estándar (porciento/100) de la serie de datos es igual a 0.13. La incertidumbre estándar está dada por:

$$
u_{R c}=S_{R c} / \sqrt{ } 6=0.13
$$

\section{Balance o presupuesto de las incertidumbres}

Para completar la tabla que muestra el presupuesto de las incertidumbres y el índice de contribución de cada variable a la incertidumbre combinada ${ }^{[9,10,15]}$, es necesario calcular los coeficientes de sensibilidad $c_{x i}$.

Estos coeficientes se calculan a partir de la derivada parcial de la función del modelo $f$ con respecto a cada una de las variables $x_{i}$ representadas en la ecuación (2). Para el cálculo de estos coeficientes se utilizó el programa de cálculo simbólico MAPLE $11^{[13]}$.

$$
c_{x i}=\frac{\partial f}{\partial x_{i}}
$$

\section{Cálculo de los coeficientes de sensibilidad $c_{x i}$ :}

Tal a como se discutió en un trabajo anterior ${ }^{[10]}$, cuando las funciones son complejas el cálculo simbólico Maple 11 simplifica el tiempo de cálculo. Además la precisión es ilimitada y no utiliza redondeos.

Esto es muy importante en el cálculo de las incertidumbres, los redondeos intermediarios pueden provocar grandes imprecisiones en el resultado final.

El redondeo y el número de cifras significativas deben aplicarse al resultado final. A continuación se detalla el cálculo para encontrar el coeficiente de sensibilidad de la variable $A x$ (área del pico cromatográfico de la aflatoxina B1 en la muestra). 
Se escribe la función $f$ en el lenguaje simbólico de Maple:

$$
f:=\left(\frac{A x}{A s}\right) *\left(\frac{C M R C * P * v 01 * v 03}{v 02 *(v 03+v 04+v 05)} * 1000\right) *\left(\frac{V 4 *(V 1+V 2) *(V 0+V s) * V_{6}}{V 5 * V 3 * V 1 * w}\right) *\left(\frac{100}{R c}\right):
$$

La derivada de la función $f$ con respecto a $A_{x}$ se escribe de la siguiente manera:

$$
\operatorname{print}\left(c_{A x}:=\frac{\partial}{\partial_{A x}} f\right)
$$

El resultado que se obtiene es el siguiente:

$$
c A x=\frac{100000 C M R C P v 01 v 03 V 4(V 1+V 2)(V 0+V s) V 6}{v 02(v 03+v 04+v 05) V 5 V 3 V 1 w A s R c}
$$

De la misma manera se evaluaron los coeficientes de sensibilidad para cada una de las variables del modelo, excepto para el parámetro $P$, que se ha tomado como constante, por no conocer su incertidumbre.

Asignando los valores a cada una de las variables que se obtienen a partir de la tabla I, se tienen los resultados numéricos de $c_{x i}$. La tabla IV muestra el presupuesto de las incertidumbres, donde se pueden ver las diferentes variables $x_{i}$, las incertidumbres, los coeficientes de sensibilidad correspondientes, el valor absoluto del producto del coeficiente de sensibilidad por la incertidumbre de $x_{i} \mathrm{y}$, por último, el cuadrado del producto de los coeficientes por las incertidumbres.

\section{Cálculo de la incertidumbre $u_{y}$}

Para calcular la incertidumbre en la determinación de aflatoxina B1 en una muestra de maní de exportación, se aplica la ley de propagación de la incertidumbre (ecuación 1) al modelo matemático representado en la ecuación (2); si se admite que no existen variables correlacionadas la expresión es la siguiente:

$$
u_{y}=\sqrt{\sum\left(c_{x i} \times u_{x i}\right)^{2}}
$$

TABLA IV. Presupuesto de las incertidumbres en la medición del contenido de aflatoxina B1 en maní de exportación.

\begin{tabular}{cccccc}
\hline Variable $x_{i}$ & Valor & $c_{x i}$ & $u_{x i}$ & $\left|c_{x i} \times u_{x i}\right|$ & $\left(c_{x i} \times u_{x i}\right)^{2}$ \\
\hline$A x$ & 12.22328 & 0.12063349 & 1.47821886 & 0.1783227 & 0.03179899 \\
$A s$ & 76.09827 & -0.01937675 & 1.10384003 & 0.02138883 & 0.00045748 \\
$C_{M R C}$ & 3.415 & 0.43178241 & 0.01443376 & 0.00623224 & $3.8841 \times 10^{-5}$ \\
$v_{0 I}$ & 1 & 1.47453693 & 0.05720036 & 0.08434405 & 0.00711392 \\
$V_{03}$ & 1 & 1.44562444 & 0.05720036 & 0.08269024 & 0.00683768 \\
$V_{02}$ & 10 & -0.14745369 & 0.02328687 & 0.00343374 & $1.1791 \times 10^{-5}$ \\
$V_{04}$ & 25 & -0.02891249 & 0.01709702 & 0.00049432 & $2.4435 \times 10^{-7}$ \\
$V_{05}$ & 25 & -0.02891249 & 0.05640609 & 0.00163084 & $2.6596 \times 10^{-6}$ \\
$V_{4}$ & 3.5 & 0.42129627 & 0.14516939 & 0.06115932 & 0.00374046 \\
$V_{I}$ & 30 & -0.01966049 & 0.0539409 & 0.0010605 & $1.1247 \times 10^{-6}$ \\
$V_{2}$ & 20 & 0.02949074 & 0.02188784 & 0.00064549 & $4.1666 \times 10^{-7}$ \\
$V_{0}$ & 200 & 0.00491512 & 0.92813358 & 0.00456189 & $2.0811 \times 10^{-5}$ \\
$V_{s}$ & 100 & 0.00491512 & 0.0369414 & 0.00018157 & $3.2968 \times 10^{-8}$ \\
$V_{6}$ & 1.6 & 0.92158558 & 0.02093419 & 0.01929265 & 0.00037221 \\
$V_{5}$ & 1.6 & -0.92158558 & 0.00585692 & 0.00539765 & $2.9135 \times 10^{-5}$ \\
$V_{3}$ & 40 & -0.03686342 & 0.21637327 & 0.00797626 & $6.3621 \times 10^{-5}$ \\
$w$ & 33.33 & -0.04423655 & 0.00763763 & 0.00033786 & $1.1415 \times 10^{-7}$ \\
$R_{c}$ & 94.78 & -0.01555747 & 0.05 & 0.00077787 & $6.0509 \times 10^{-7}$ \\
\hline
\end{tabular}


El cálculo se vuelve simple si se suma la última columna de la tabla IV y se extrae la raíz cuadrada. De esta manera se tiene el siguiente valor:

$$
u_{y}=\sqrt{0.05045943}=0.224632
$$

La incertidumbre estándar relativa como porcentaje (ISR\%) puede calcularse según la expresión siguiente:

$$
I S R \%=\frac{u_{y}}{y} \times 100
$$

Donde $y=f$ y se obtiene sustituyendo el valor de las variables en el modelo matemático de la ecuación (2). De esta forma la $I S R \%$ es:

$$
I S R \%=\frac{0.224632}{1.4745369} \times 100=15.2 \%
$$

Si se toma en cuenta que a niveles de trazas las precisiones pueden alcanzar hasta el $20 \%$ de incertidumbres relativas ${ }^{[26]}$, se puede afirmar que los niveles de precisiónen el presentetrabajo sonaceptables. No obstante, se hace necesario realizar un análisis de las contribuciones de cada una de los componentes de la incertidumbre para ver la factibilidad de reducir la incertidumbre estándar relativa. El diagrama de Pareto con una significancia $90: 10^{[10]}$, es de gran utilidad para actuar sobre las variables que tienen mayor influencia en la incertidumbre del mensurando.

Índice de contribución de los componentes de la incertidumbre y diagrama de Pareto

La contribución de cada variable a la incertidumbre del contenido de aflatoxina B1 en la muestra se evalúa con el índice de contribución expresado en porcentaje según la ecuación siguiente:

$$
\text { Ind } \%=\frac{\left|c_{x i} u_{x i}\right|}{\sum\left|c_{x i} u_{x i}\right|}(100)
$$

Esta ecuación es más rigurosa que la utilizada en trabajos anteriores ${ }^{[10]}$; tiene mayor precisión en el cálculo del índice de contribución para establecer los criterios de las variables que más influyen en la incertidumbre del mensurando $y$. Es equivalente al índice de contribución establecido en la guía de la expresión de la incertidumbre de la EURACHEM ${ }^{[9]}$. Los resultados del cálculo realizado con la ecuación (5) se presentan en la tabla $\mathrm{V}$.

En esta tabla se presentan también los resultados de los índices acumulativos (Ind Acum). A partir de los datos de esta tabla se construye el Diagrama de Pareto representado en la figura 4.
Tabla V. Índice de contribución de los componentes de la incertidumbre de los resultados del contenido de aflatoxina B1 en maní.

\begin{tabular}{ccc}
\hline Variable & Ind $\%$ & Ind $_{\text {Acum }}$ \\
\hline$A_{x}$ & 37.2 & 37.2 \\
$v_{01}$ & 17.6 & 54.7 \\
$V_{03}$ & 17.2 & 72 \\
$V_{4}$ & 12.7 & 84.7 \\
$A_{s}$ & 4.5 & 89.2 \\
$V_{6}$ & 4 & 93.2 \\
$V_{3}$ & 1.7 & 94.8 \\
$C_{m}$ & 1.3 & 96.1 \\
$V_{5}$ & 1.1 & 97.3 \\
$V_{0}$ & 1 & 98.2 \\
$V_{02}$ & 0.7 & 98.9 \\
$V_{05}$ & 0.3 & 99.3 \\
$V_{1}$ & 0.2 & 99.5 \\
$R_{c}$ & 0.2 & 99.7 \\
$V_{2}$ & 0.1 & 99.8 \\
$V_{04}$ & 0.1 & 99.9 \\
$w$ & 0.1 & 100 \\
$V_{s}$ & 0 & 100 \\
\hline
\end{tabular}

En el diagrama de Pareto se puede ver que las variables que contribuyen significativamente en la incertidumbre de $y$, son las siguientes: $A_{x}, v_{01}, v_{03}, V_{4}$ y $A s$.

La incertidumbre en la medición del área del pico cromatográfico de la aflatoxina B1 en la muestra de maní $\left(A_{x}\right)$, tiene una contribución del $37 \%$, seguido de las variables $v_{01}$ y $v_{03}$ con 18 y 17 por ciento cada una, las que representan las incertidumbres en las mediciones de las alícuotas de solución de referencia certificada utilizadas en la preparación de la concentración del patrón de la aflatoxina.

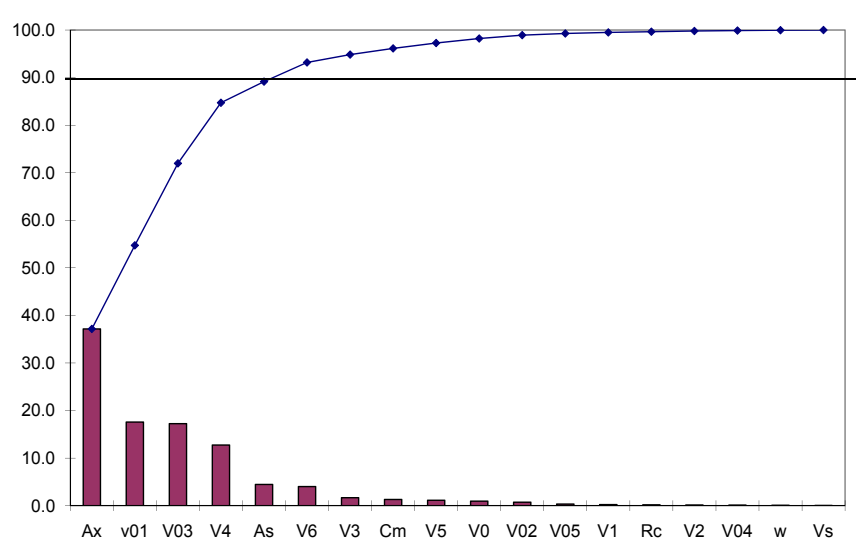

Figura 4. Diagrama de Pareto representando la contribución de las diferentes variables que contribuyen a la incertidumbre en la medición de aflatoxina B1 en maní de exportación. 
La incertidumbre en la medición del volumen de cloroformo $V_{4}$ contribuye en $13 \%$ y la medida en el área de pico cromatográfico de la solución patrón o de referencia de la aflatoxina contribuye en un $5 \%$. Estos cinco componentes representan el $90 \%$ de la contribución acumulativa en la incertidumbre del mensurando $y$. La variable sobre la que hay que actuar primero es en la incertidumbre en el área de pico de muestra $u_{A x}$.

Para ello, es necesario estudiar el aporte de las desviaciones estándares de los tres componentes que afectan la medición de esta variable: precisión intermedia en la lectura de las áreas de pico de la muestra de referencia interna, la precisión del ruido de fondo y la precisión en la deriva de la línea de base de la respuesta del HPLC. Al graficar las desviaciones estándares de estos tres componentes se tiene la figura 5.

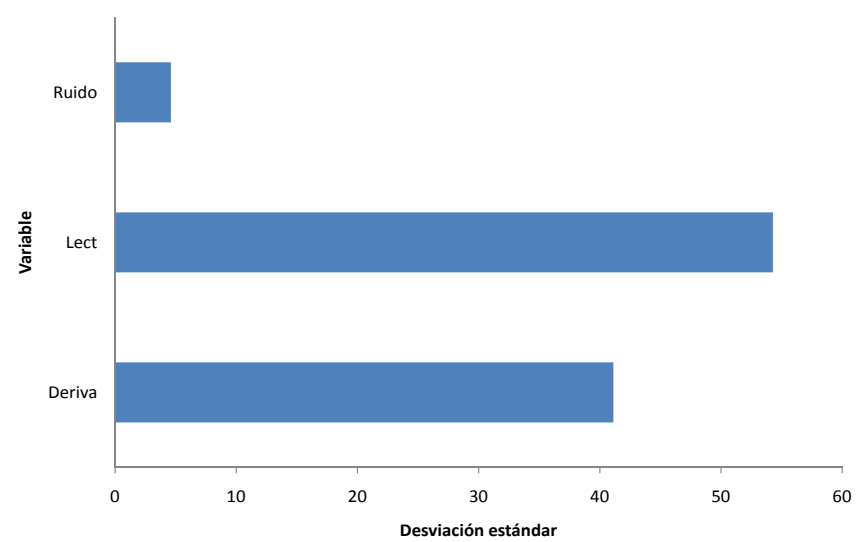

Figura 5. Diagrama de barra de las desviaciones estándares para las variables ruido, lectura de áreas de picos y deriva de la señal de fondo del HPLC en la medición del área de pico cromatográfico de la aflatoxina en la muestra.

Se puede ver que las lecturas del área de pico de la aflatoxina B1 en la muestra es la que tiene mayor influencia en la incertidumbre del mensurando. Seguido de la deriva de la señal de fondo del instrumento. Para reducir estas incertidumbres será necesario estudiar las causas de la alta dispersión.

Con respecto al área de pico de la aflatoxina B1 en la muestra, la dispersión en las mediciones puede estar afectada por la heterogeneidad de la muestra, tanto a nivel del contenido en aflatoxina como en los componentes de la matriz; también pudiera estar afectada por las condiciones de almacenamiento y/o estabilidad de la muestra de negativa (cero contenido de aflatoxina), a partir de la cual se prepara la muestra de referencia interna para el control mensual del método.
Se podría hacer un estudio más detallado en estos aspectos con el fin de ver la factibilidad de reducir la dispersión. En cuanto al efecto de la incertidumbre en la deriva, será necesario comparar con los resultados del sistema de cualificación del equipo para verificar si hay conformidad con los niveles especificados por el fabricante. En caso de no haber coincidencia, será necesario realizar los ajustes experimentales necesarios para reducir la incertidumbre en la deriva.

Una vez lograda la reducción de la incertidumbre en la medición del área de pico de la muestra, $u_{A x}$, se deberán buscar volumétricos de mejor calidad para realizar las mediciones de los volúmenes $v_{01}, v_{03}$ y $V_{4}$ utilizados en la preparación de la solución de referencia y en la adición de cloroformo (solvente extractor) a la solución acuosa de la muestra.

Por último, es necesario tratar de reducir la incertidumbre en la medición del área de pico del estándar $\left(A_{s}\right)$; se deberá estudiar la precisión en las lecturas, el ruido y la deriva. De lo anterior se puede deducir, que si se consideran solamente las incertidumbres de las 5 primeras variables del diagrama de Pareto (figura 4) se puede estimar la incertidumbre del mensurando y con una aproximación de más del 99\%.

El detalle se discute a continuación. La incertidumbre calculada por la raíz cuadrada de la suma de los cuadrados del producto de los coeficientes de sensibilidad por las incertidumbres de los cinco componentes que contribuyen en un $90 \%$ a la incertidumbre del mensurando, está dada por la siguiente expresión:

$$
u_{y}=\sqrt{\left(c_{A x} \times u_{A x}\right)^{2}+\left(c_{v 01} \times u_{v 01}\right)^{2}+\left(c_{v 03} \times u_{003}\right)^{2}+\left(c_{V 4} \times u_{V 4}\right)^{2}+\left(c_{A S} \times u_{A S}\right)^{2}}
$$

Sustituyendo los valores correspondientes de la columna 6 de la tabla IV, se obtiene:

$u_{y}=\sqrt{0.031798986+0.000849245+0.000816268+0.00374046+0.00045748}=0.22349167$

El grado de aproximación se calcula de la siguiente manera:

$$
\left(1+\frac{0.223492-0.224632}{0.224632}\right) \times 100=99.5 \%
$$

Con lo anterior se verifica que no es necesario tomar todos los componentes para evaluar la incertidumbre, se pueden tomar solamente los cinco componentes que constituyen el $90 \%$ de la contribución, de acuerdo al diagrama de Pareto.

\section{Estimación del intervalo de confianza}

El intervalo de confianza del contenido de aflatoxina B1 en la muestra de maní del presente trabajo, considerando un factor de cobertura $k=2$, probabilidad del $95.45 \%$ y 
tomando en cuenta el número de cifras significativas correcto, se calcula de la siguiente manera:

$$
Y=y \pm U=1.4745 \pm 2 \times 0.2246
$$

$\mathrm{Y}=(1.47 \pm 0.45) \mathrm{ng} / \mathrm{g}$ de aflatoxina $\mathrm{B} 1$ en la muestra de maní.

Donde $U$ es la incertidumbre expandida. La precisión de la medición puede estimarse evaluando la incertidumbre expandida relativa ${ }^{[10]}$ (IER) expresada en porcentaje:

$$
I E R \%=\frac{U}{y} \times 100=30 \%
$$

Mediciones a niveles de trazas, del orden de $1 \mathrm{ng} / \mathrm{g}$, pueden alcanzar precisiones mayores del $30 \%{ }^{[26]}$, esto significa que el valor de la incertidumbre encontrada en el presente estudio es aceptable. No obstante, es necesaria la implementación de mejoras para reducir más la incertidumbre.

\section{CONCLUSIONES}

Aplicando las directrices generales de la guía GUM ISO 1995 y EURACHEM, se pudo evaluar la incertidumbre en la determinación de la concentración de aflatoxina B1 (el mensurando) por HPLC-FD en una muestra de maní de exportación. Se utilizó el programa de cálculo simbólico MAPLE 11 para calcular los coeficientes de sensibilidad de las diferentes variables que influyen en la estimación de la incertidumbre del mensurando.

Se hizo un estudio de la contribución de los componentes de la incertidumbre a través del diagrama de Pareto, encontrándose que los cinco componentes a tomar en cuenta para el cálculo son la incertidumbre de las áreas de pico cromatográfico de la muestra y la solución estándar, los volumétricos utilizados en la preparación de la solución de referencia y el volumen de cloroformo utilizado como solvente extractor de aflatoxinas.

El intervalo de confianza encontrado fue de $(1.47 \pm$ 0.45 ) $\mathrm{ng} / \mathrm{g}$ con un nivel de confianza del $95.45 \%$. Si se admite que la muestra es representativa del lote a exportar y las especificaciones del comprador establece que el contenido de aflatoxina $B_{1}$ debe ser menor o igual $1.5 \mathrm{ng} / \mathrm{g}$, se puede afirmar que la muestra en cuestión cumple con las especificaciones establecida por el comprador.

Es importante señalar, que uno de los problemas principales en este tipo de ensayos es la heterogeneidad de la muestra. Puede ser que muy pocos granos de maní estén afectados frente a una gran cantidad que representa un lote de producción.
Los cuidados en el almacenamiento y el transporte son unos de los puntos claves a controlar para evitar la contaminación con el hongo que produce la micotoxina.

\section{RECONOCIMIENTO}

Los autores agradecen al Laboratorio SER AGRO, S.A. por hacer posible la publicación del presente estudio, realizado dentro del contexto de la mejora continua que el laboratorio lleva a cabo para mantener la Acreditación que la Oficina Nacional de Acreditación (ONA- MIFIC) le ha otorgado desde el año 2001.

\section{REFERENCIAS BIBLIOGRAFICAS}

1. SERGIO QUISPE y SUSANA REVOLLO. (2001), "Estudios de contaminación con aflatoxinas producidas por Aspergillus flavus en la castaña y el maní", Investigadores Asociados. Unidad Biología Molecular - Inst. SELADIS - Univ. Mayor de San Andrés, Bolivia. Disponible en: http://www.engormix. com/s_articles_view.asp?art=438\&AREA=MYC.

2. a) URREGO NOVOA J.R. y Díaz, G.J., (2006), "Aflatoxinas: Mecanismos de toxicidad en la fitología del cáncer hepático celular", Rev Fac. Med. Univ. Colomb., Vol. 54, 2, 116; b) BODEY, G. P. y S. VARTIVARIAN, (1989), Aspergillosis. Eur. J. Clin. Microbiol. Infect. Dis. 8:413-437.

3. BHAT RV, VASATHI S., 1999, "Contaminación por Micotoxinas de Alimentos y Piensos", Tercera Conferencia Internacional FAO/OMS/PMA sobre Micotoxinas, Túnez. Disponible en http://www.fao. org/docrep/meeting/005/W5122S/w5122s0j.htm.

4. ILAC-G8 (1996). "Guidelines on Assessment and Reporting of compliance with specification".

5. DESIMONI, E. y BRUNETTI, B. (2006), "Considering uncertainty of measurement when assessing compliance or non-compliance with reference values given in compositional specifications and statutory limits: a proposal", Accred Qual Assur, 11, 363-366.

6. BIPM, IEC, IFCC, IUPAC, OIML (1995). Guide for to the Expression of Uncertainty in Measurement (GUM), ISO, Ginebra.

7. NIST (1993). "Guidelines for evaluation and expressing the uncertainty of NIST measurement results", Gaithersburg, USA. 
8. W.A. SCHMID y R.J. LAZOS. (2000), "Guía para estimar la incertidumbre de la medición", CENAM, México.

9. EURACHERM/CITAC GUIDE, (2000), Quantifying Uncertainty in Analytical Chemistry, 2da edición, UK.

10. DELGADO G y NAGEL B. (2008) "Un experimento sencillo para evaluar la incertidumbre siguiendo la guía GUM ISO 1995 y utilizando el cálculo simbólico MAPLE 11.0", Universitas UNAN-León, Vol 1, 2-1926.

11. ISO/IEC 17025(ES) (2005). "Requisitos Generales para la Competencia de los Laboratorios de Ensayo y de Calibración", Norma Internacional, Ginebra, Segunda Edición.

12. ONA, DOC-ONA-007-04, "Reglamento de Acreditación de la OFICINA NACIONAL DE ACREDITACIÓN, Dirección de Tecnología, Normalización y Metrología", Ministerio de Industria y Fomento de Nicaragua, MIFIC. Disponible en http//www.mific.ni/dirmercados/docsONA/ DOCONA-007-0. Acceso el 11/2008.

13. a) CHAR B. W. (1992), Manual Maple V, SpringerVerlag, New York; b) Maple 11.0, Tutorial, software.

14. NIST NTN 1297, append A. Law of propagation uncertainty. http//physic.nist.gov/pubs/guideline/ appa.html. Acceso el 10/10/2008.

15. KIMOTHI, S.K., (2002), The Uncertainty of Measurements, ASQ Quality Press, Wisconsin , pag. 205.

16. HERRADOR, M.A., GONZÁLEZ G.A., (2004), "Evaluation of Measurement uncertainty in Analytical Assayes by means of Monte Carlo Simulation", Talanta, Vol. 64, 6, 415-422.

17. OGREN P., DAVIS B y NICK G., (2001), "Curve fitting, confidence intervals and envelopes, correlations, and Monte Carlo visualization for multilinear problems in chemistry", J. C hem. Edu., Vol. 78, 6, 827-836.

18. MEIER P.C. y ZUND R. E., (2000), Statistical Methods in Analytical Chemistry, 2th Ed., John Wiley and Sons Inc., New York, pag. 163.
19. SAOSA-01, (2007), Procedimiento para la determinación de Aflatoxinas por HPLC, Laboratorio SER-AGRO, Chinandega.

20. SAOSA-PT-04, (2006), Reporte de validación del análisis de aflatoxinas por HPLC, Laboratorio SERAGRO, Chinandega.

21. ICH (International Conference on Harmonisation of Technical Requirements for Registration of Pharmaceutical for Humans), (2005). Validation of Analytical Procedures Text and Methodology Q2(R1).

22. SAOSA-PT-12, (2007), Procedimiento de control de la validez en rutina del análisis de aflatoxinas pór HPLC, Laboratorio SER-AGRO, Chinandega.

23. MANUAL HPLC, (2000), Evaluación de la Idoneidad del Sistema, Agilent Thecnologies, pag. 250.

24. SAOSA-PT-16, (2008), Procedimiento de evaluación de la incertidumbre, Laboratorio SER-AGRO, Chinandega.

25. DEAN J.A., (1979), Lange's Handbook, Mc GrawHill, Inc., 12a Ed., New York, pág. 10-127.

26. FEINBERG M, (1996), La validation de méthods d'analyse, Masson, París, pag. 117. 